\title{
Case Report \\ Extensive Epidermal Skin Loss Secondary to HSV Type One: Neonatal Management Challenges
}

\author{
Rebecca J Calthorpe (D, ${ }^{1}$ Emma Spencer $\left(D,{ }^{1}\right.$ Jane C Ravenscroft, ${ }^{2}$ Ting S Tang, \\ Anna E Martinez, ${ }^{3}$ and Anjum Deorukhkar ${ }^{1}$ \\ ${ }^{1}$ Department of Neonatology, Nottingham University Hospitals NHS Trust, Nottingham, UK \\ ${ }^{2}$ Department of Dermatology, Nottingham University Hospitals NHS Trust, Nottingham, UK \\ ${ }^{3}$ Department of Dermatology, Great Ormond Street Hospitals, London, UK \\ Correspondence should be addressed to Emma Spencer; emma.spencer4@nhs.net
}

Received 29 August 2019; Accepted 5 November 2019; Published 5 December 2019

Academic Editor: Vjekoslav Krzelj

Copyright (c) 2019 Rebecca J Calthorpe et al. This is an open access article distributed under the Creative Commons Attribution License, which permits unrestricted use, distribution, and reproduction in any medium, provided the original work is properly cited.

\begin{abstract}
We describe a rare case of a preterm neonate presenting at birth with extensive epidermal skin loss of over $90 \%$ due to disseminated herpes simplex virus type one infection. Differential diagnosis included aplasia cutis and epidermolysis bullosa. Serum PCR and mouth swabs confirmed HSV type one, and the patient required three weeks of treatment with intravenous aciclovir, followed by oral aciclovir. We describe the management challenges and give practical solutions applicable to the care of a neonate presenting with widespread skin loss due to any aetiology.
\end{abstract}

\section{Introduction}

This report describes a rare case where a thirty-week gestation neonate had over $90 \%$ epidermal skin loss at delivery due to disseminated, transplacentally acquired herpes simplex virus (HSV) type one. This resulted in complex neonatal care that was atypical to a baby of this gestation. Typically, the neonatal course for an infant born at this gestation in the United Kingdom (UK) would involve a short period of respiratory support and establishment of central venous access to administer parenteral nutrition until feeds are introduced. They would receive a course of antibiotics, may require phototherapy to manage jaundice, and will routinely need cranial ultrasound scans and screening for retinopathy of prematurity. Survival rates in the UK for a thirty-week gestation neonate are $96 \%$ [1]. This markedly contrasts survival rates in paediatric burn patients where those with $90 \%$ skin damage have $50 \%$ mortality at one year after injury [2]. Consequently, the extent of skin loss experienced in this case required a tailored management approach.

In July 2019, the British Paediatric Surveillance Unit (BPSU) launched a two-year study that aims to assess the treatment burden of HSV in neonates less than 90 days, in order to inform detection and management decisions [3]. This will be beneficial in the treatment of neonatal HSV in the future, particularly as the disease has devastating consequences. Currently, there is limited literature available to guide the management of widespread skin loss due to HSV. Therefore, given the success of this case despite its complexity, we provide information on the diagnostic challenges and the individualised care employed. We also give practical treatment strategies for the management of a neonate with widespread skin loss due to HSV that are applicable to any aetiology of widespread epidermal skin loss in a neonate (Table 1).

\section{Case Description}

A thirty-week gestation preterm neonate (birth weight: $1250 \mathrm{~g}$ ) was born via normal vaginal delivery to a healthy primip mother in a hospital where there was tertiary level neonatal care. There had been spontaneous rupture of membranes 48 hours prior to delivery, and a background history of a small antepartum haemorrhage at twenty-nine 
TABLE 1: Table outlining the key management points when treating a neonate with widespread skin loss.

\begin{tabular}{|c|c|}
\hline \multicolumn{2}{|r|}{ System-based considerations when managing a neonate with widespread skin loss } \\
\hline Respiratory & $\begin{array}{l}\text { Invasive ventilation can allow the clinician to optimise analgesia administration and avoid further skin } \\
\text { damage to the head and face. }\end{array}$ \\
\hline Intravenous access & $\begin{array}{l}\text { Intravenous access may be difficult to obtain and secure; maximise the duration of central line access } \\
\text { with careful monitoring. Umbilical access should be secured to the umbilical cord and not the skin. UAC } \\
\text { access facilitates blood sampling as heel prick blood sampling should be avoided. }\end{array}$ \\
\hline Monitoring & $\begin{array}{c}\text { Tailor how routine observations are performed and do not attach electrodes to the skin or use skin } \\
\text { temperature probes. An oxygen saturation probe can be used to monitor the heart rate, and blood } \\
\text { pressure measurements can be obtained via a UAC. }\end{array}$ \\
\hline Fluids & $\begin{array}{l}\text { There will be excessive fluid loss via the skin; therefore, fluid balance monitoring should be documented } \\
\text { hourly to aid decisions on fluid management. }\end{array}$ \\
\hline Nutrition & $\begin{array}{l}\text { Nutrition is important to promote skin healing and should be considered early. If central venous access } \\
\text { has been obtained, parenteral nutrition can be prescribed until milk feeds are commenced. }\end{array}$ \\
\hline Analgesia & $\begin{array}{l}\text { Hourly monitoring of pain using a classified pain scoring system will direct pain management. Rapid } \\
\text { escalation of analgesia with drugs not typically prescribed in neonates may be needed; therefore, expert } \\
\text { pharmacy advice should be accessed where available. }\end{array}$ \\
\hline Jaundice & $\begin{array}{l}\text { In this case, phototherapy was implemented effectively to treat jaundice with no obvious exacerbation to } \\
\text { the skin. }\end{array}$ \\
\hline Neurology & $\begin{array}{l}\text { The clinician should be aware of the neurological complications related to HSV infection. If a lumbar } \\
\text { puncture cannot be performed, liaise with a virologist to discuss treatment options. Cranial ultrasound } \\
\text { and MRI scans should be used to aid diagnosis of neurological morbidity. }\end{array}$ \\
\hline Microbiology & $\begin{array}{l}\text { Consider HSV infection in a neonate presenting with widespread skin loss and obtain samples for viral } \\
\text { PCR. Administer intravenous aciclovir early. There is an increased risk of infection; therefore, have a low } \\
\text { threshold for commencing antimicrobial treatment if there are signs of infection. }\end{array}$ \\
\hline Place of care & $\begin{array}{l}\text { Transfer to a tertiary neonatal unit is advisable where all members of the MDT are available to offer } \\
\text { expert review and treatment input. }\end{array}$ \\
\hline Communication & $\begin{array}{l}\text { Provide clear communication with the family at all stages of admission about treatment decisions and } \\
\text { outcome expectations. }\end{array}$ \\
\hline \multicolumn{2}{|r|}{ Specific skin considerations when managing a neonate with widespread skin loss } \\
\hline Handling & Ensure careful handling to avoid skin friction; handling techniques can be taught by the EB team. \\
\hline Dressings & $\begin{array}{c}\text { Give adequate analgesia } 15 \text { to } 30 \text { minutes prior to dressing application. Apply dressings to areas of raw } \\
\text { skin, in this case Mepilex Transfer was applied, held in place with Tubifast bandage. Cling film can be } \\
\text { used if no other dressings are immediately available. }\end{array}$ \\
\hline Emollients & $\begin{array}{l}\text { Apply emollients such as Dermol } 500 \text { and } 50: 50 \text { Paraffin lotion to the skin. Emollients such as } 50: 50 \\
\text { Paraffin lotion can be applied to the fingers prior to handling. }\end{array}$ \\
\hline
\end{tabular}

UAC: umbilical arterial catheter; PCR: polymerase chain reaction.

weeks. Prior to this, the pregnancy had been uncomplicated with a normal antenatal scan performed at twenty weeks gestation. There was a known maternal history of Group B Streptococcal infection and a past history of oral herpes simplex infection in both parents but no known genital lesions. At delivery, the infant had extensive epidermal skin loss of over $90 \%$ with sparing of the soles of the feet, fingers, genital area, and central face (Figure 1(a)). The extent of skin loss was estimated using the Mersey Burns calculator [4]. Widespread skin loss resulted in complex care beyond that usually required for an infant born at this gestation.

2.1. Respiratory. He was intubated and given $200 \mathrm{mg} / \mathrm{kg}$ surfactant in the delivery suite due to poor respiratory drive. He subsequently required minimal invasive ventilation; however, extubation to noninvasive support was impeded by concerns that the CPAP face mask would exacerbate skin damage. He was also on high-dose opiates for pain management with the associated risk of respiratory depression. He therefore remained intubated until day seven of life, at which point he was extubated straight into head box oxygen.

2.2. Monitoring and Intravenous Access. A fundamental issue in his care was that intravenous access was difficult to obtain and secure. Extensive skin loss also hindered monitoring of observations, normally done via blood pressure cuffs, saturation probes, and electrocardiogram leads. Heart rate and blood pressure were monitored invasively via an umbilical arterial catheter (UAC), which avoided further skin damage and facilitated blood sampling. Unfortunately, the umbilical venous catheter (UVC) was not optimally placed or secured, hence required reinsertion on day three and again on day seven to attempt better placement. Long-line insertion in the limbs was not feasible; consequently the umbilical venous catheter was used for an extended period beyond what was considered as acceptable routine practice on the neonatal unit. A long line was later inserted in a scalp vein due to 

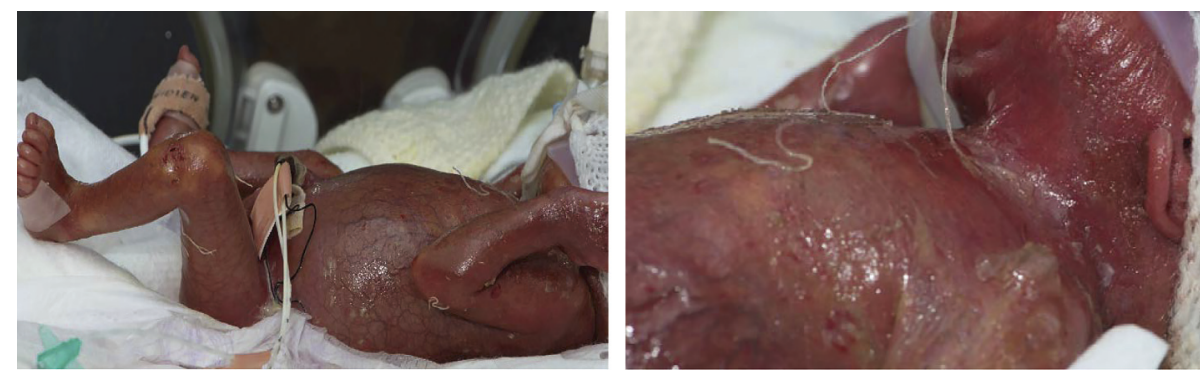

(a)
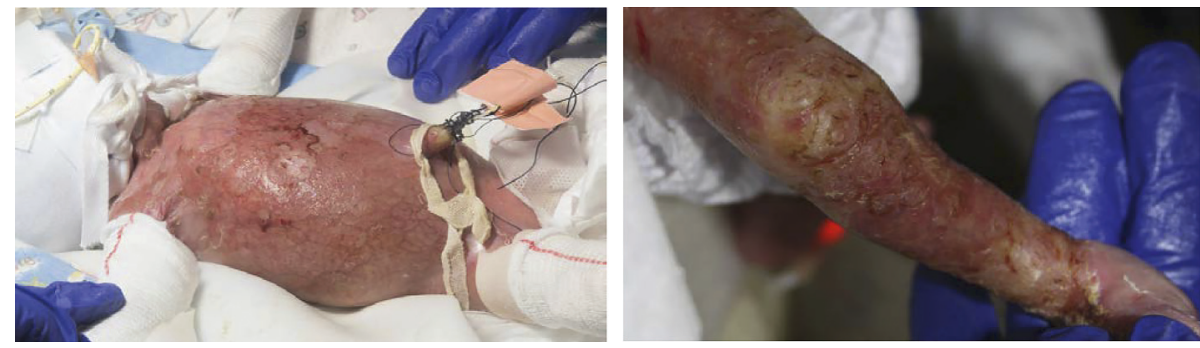

(b)
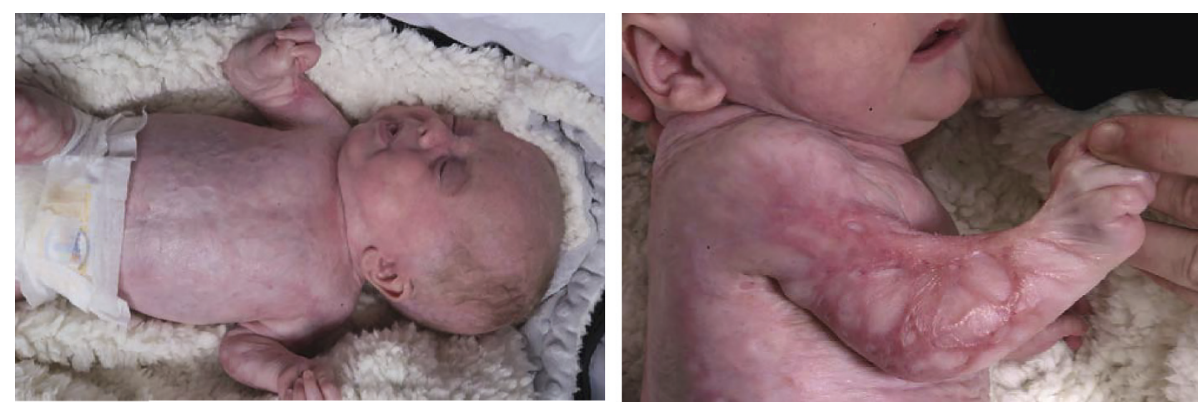

(c)

FIGURE 1: Images of the neonate following presentation at birth (a), at one week of age with some visible skin reepithelisation (b), and at four months of age following the development of cribiform scarring and contractures (c).

relative skin sparing in this area. In total, the UVC and UAC remained in situ for ten days. During this time, routine monitoring included twice daily examination of the umbilicus and hourly documentation of the fluid infusion volume and pressure. The UVC tip from the replaced umbilical venous catheters was sent for microbiology to assess the likelihood of possible secondary central line infection (Table 2).

2.3. Fluid and Nutrition. He required an initial $40 \mathrm{ml} / \mathrm{kg}$ fluid bolus and rapid escalation of fluids to a maximum of $200 \mathrm{ml} / \mathrm{kg} /$ day to account for high insensible fluid loss from the skin, associated hypotension, and to avoid acute prerenal injury. He was also recurrently anaemic and thrombocytopenic. Local hospital guidelines detailed the transfusion thresholds for both red cell and platelet transfusions [5, 6]. A transfusion threshold of haemoglobin below $120 \mathrm{~g} / \mathrm{l}$ for a ventilated preterm and platelets $<30 \times 109 / \mathrm{L}$ for a sick preterm were employed and administered at a volume of $20 \mathrm{ml} / \mathrm{kg}$ and $10 \mathrm{ml} / \mathrm{kg}$, respectively. Parenteral nutrition was administered via the suboptimally placed umbilical venous catheter, and trophic feeds of expressed breast milk were commenced at the earliest opportunity. He remained on
TABLE 2: Table outlining the results of key microbiology results according to the day the test was taken and organised by the type of microbiological investigation.

\begin{tabular}{lcc}
\hline Day & Microbiological investigation & Result \\
\hline 0 & Blood PCR & HSV1 \\
9 & Blood PCR & Negative \\
16 & Blood PCR & Low levels of HSV1 \\
19 & Blood PCR & Negative \\
24 & Blood PCR & Negative \\
\hline 0 & Skin swab & HSV1 \\
16 & Skin swab & Negative \\
\hline 0 & Blood culture & Negative \\
4 & Blood culture & Enterococcus \\
13 & Blood culture & Negative \\
\hline 3 & UVC tip & Enterococcus \\
7 & UVC tip & Enterococcus \\
10 & UVC tip & Enterococcus \\
10 & UAC tip & Enterococcus \\
\hline
\end{tabular}

trophic feeds for nine days due to the risk of necrotising enterocolitis in a preterm with umbilical lines in situ and on high-dose opiates. Feeds were increased quickly from day 
nine, and full feeds at $165 \mathrm{ml} / \mathrm{kg} /$ day of expressed breast milk were established by day twelve.

2.4. Jaundice and Phototherapy. Within the first twenty-four hours, serum bilirubin was measured on the exchange transfusion line on the National Institute for Health and Care Excellence (NICE) treatment threshold graphs. The efficacy of phototherapy in a neonate with extensive skin loss was unknown, but despite this conventional blue light phototherapy was employed at an intensified level using three separate overhead lights due to the risk of kernicterus. Once the serum bilirubin was below the exchange transfusion line, the use of phototherapy was moderated due to concerns that it would exacerbate skin damage and insensible fluid loss. A pragmatic approach was taken with six hourly serum bilirubin measurements used to aid decisions on the duration of phototherapy. Phototherapy was stopped by day two, when the bilirubin level reached the phototherapy treatment line. Overall, he had a good response to treatment with no obvious skin exacerbation.

2.5. Pain. This was one of the most challenging aspects of care. Pain was assessed hourly using the Pain Assessment Tool (PAT), which uses a combination of physiological observations, behavioural parameters, and nursing perception to give a score out of twenty for pain [7]. Due to consistently high pain scores, exacerbated by spontaneous movement and handling, he required rapid escalation of analgesia. The analgesia prescribed was that typically administered in the paediatric intensive care setting. Use of ketamine and fentanyl infusions up to a maximum dose of 100 micrograms $/ \mathrm{kg}$ and 5 micrograms $/ \mathrm{kg}$, respectively, helped achieve better pain control. Additional intravenous boluses were administered before routine cares and during dressing changes.

2.6. Microbiology. Serum PCR and mouth and skin swabs taken on admission to the neonatal unit were positive for herpes simplex virus type one and remained positive until day nineteen of life (Table 2). There is a high risk of neurological complications, including meningitis and encephalitis in HSV infection. Lumbar puncture could not be performed due to extensive skin loss on the back. Instead, close monitoring of viral load based on serum PCR was performed on advice of Microbiology. Twenty-one days of intravenous aciclovir was completed, followed by a sixmonth course of oral aciclovir at a dose of $300 \mathrm{mg} / \mathrm{m}^{2} /$ day. An added complication from day three was infection with Enterococcus grown from the UVC tip and blood cultures (Table 2); this was successfully treated with a seven-day course of intravenous vancomycin.

2.7. Neurology. Mild to moderate ventriculomegaly was present on serial cranial ultrasound scans, likely posthaemorrhagic in origin. Subsequent MRI brain performed at corrected term gestation was normal.
2.8. Ophthalmology. Routine screening for retinopathy of prematurity was performed in view of multiple risk factors, and this was normal at the time of discharge from hospital at $37+5$ weeks corrected gestation.

2.9. Multidisciplinary Team (MDT). There was extensive MDT involvement throughout admission. His presentation was not classical for one condition. The national epidermolysis bullosa (EB) team based at Great Ormond Street Hospital gave immediate telephone support, followed by an outreach service from Birmingham, who were geographically closer. They performed a skin biopsy, provided dressings advice, and supported the family during the initial diagnostic period. Severe subtypes of epidermolysis bullosa were excluded with the skin biopsy, and on day seven he was noted to have reepithelisation of skin (Figure 1(b)). Dermatology supported with diagnostic and treatment advice, in addition to liaising with the Plastics team on skin dressings. His skin was managed with dressings, emollients, and Octenisan wash (Table 1). Both teams played a role in the education of the neonatal team and parents around skin treatment. Once the skin started to reepithelise, he developed contractures at multiple joints with widespread cribriform scarring. This was managed by Plastics and Physiotherapy who promoted mobilisation and the application of splints. The success of this case is owed to the support of the dedicated MDT in a tertiary centre with close involvement of the EB team.

2.10. Long Term. At 34 weeks corrected gestation, he was transferred to the local neonatal unit for ongoing care. He was subsequently discharged home at $37+5$ weeks corrected gestation with a plan for long-term follow-up with a general paediatrician. At this point, there were no further concerns with acute infection and he continued on the planned sixmonth course of oral aciclovir. His skin was managed with regular application of Dermol and Paraffin lotion on advice of the local Dermatology team. At the time of submitting this case report for publication, he was four months of age and the main ongoing issue was related to extensive cribriform scarring and skin contractures (Figure 1(c)). This was being managed by outpatient Physiotherapy and the Plastics team who were considering the need for skin grafting long term.

\section{Discussion}

Congenital HSV infection has an estimated incidence from $1: 1400$ to $1: 30000$ deliveries [8]. It can result in long-term neurodisability or death with a mortality rate of $50 \%$ [8]. Over $75 \%$ of cases are caused by HSV type two and are acquired peripartum. This case of transplacentally acquired intrauterine infection with HSV type one is extremely rare. Intrauterine HSV1 infection has an increased risk of preterm labour, as seen in this case, and intrauterine growth restriction. There are three well-described presentations of HSV infection: disseminated disease (20\%); central nervous system disease (30\%); and skin, eyes, and mouth disease (45\%) [8]. Cutaneous lesions are the most common 
presentation in up to two thirds of affected neonates, either in isolation or in association with central nervous system disease or disseminated disease $[8,9]$. Types of cutaneous presentations include vesicles, bullous lesions, erosions, ulcers, pustules, erythema, plaques, and hypopigmented scarring. To our knowledge, widespread extensive skin loss, as seen in this case, has not been reported in the literature [9].

The differential diagnosis at presentation included epidermolysis bullosa, aplasia cutis, or skin loss secondary to infection. Assessment of associated congenital abnormalities may give a clue to a genetic diagnosis; however, there should be a high clinical suspicion of HSV and early empirical treatment with intravenous aciclovir. Viral and bacterial skin swabs and serum samples should be taken for PCR prior to commencing antimicrobial treatment. There are two national Paediatric EB services in the United Kingdom (London and Birmingham) who can be contacted regarding skin care management and will give advice about any child with skin fragility. They also have written clinical guidelines detailing skin care, which were employed in the management of this neonate [10].

Given the rarity of this presentation and the difficulties in management, this case report has provided practical solutions for the additional treatment challenges faced when caring for a neonate with extensive skin loss of any aetiology (Table 1). Early recognition, prompt referral to the national Paediatric EB team, and tertiary neonatal intensive care are essential. MDT involvement is paramount and can be credited with the successful management of this unusual presentation.

\section{Acronyms}

ECG: Electrocardiogram

EB: Epidermolysis bullosa

HSV1: Herpes simplex virus type one

MDT: Multidisciplinary team

NICE: National Institute for Health and Care Excellence

$\mathrm{PN}$ : Parenteral nutrition

PCR: Polymerase chain reaction

UAC: Umbilical arterial catheter

UVC: Umbilical venous catheter.

\section{Conflicts of Interest}

There are no conflicts of interest or financial support for any of the authors listed.

\section{Authors' Contributions}

RJC and ES contributed equally to the writing of this manuscript (joint first authors), with all authors involved in the review process.

\section{Acknowledgments}

The authors would like to thank the family for their consent for the publication of this case and allowing them to raise awareness of this rare presentation of neonatal herpes simplex.

\section{References}

[1] Office for National Statistics, Gestation-Specific Infant Mortality: 2012, Office for National Statistics, London, UK, October 2014, https://www.ons.gov.uk/peoplepopulationandcommunity/ healthandsocialcare/causesofdeath/bulletins/pregnancyandet hnicfactorsinfluencingbirthsandinfantmortality/2014-10-15.

[2] R. Kraft, D. N. Herndon, A. M. Al-Mousawi, F. N. Williams, C. C. Finnerty, and M. G. Jeschke, "Burn size and survival probability in paediatric patients in modern burn care: a prospective observational cohort study," The Lancet, vol. 379, no. 9820, pp. 1013-1021, 2012.

[3] British Paediatric Surveillance Unit, Royal College of Paediatrics and Child Health (RCPCH), Neonatal Herpes Simplex Vius Disease in Infants Less Than 90 Days of Age. 2019, Royal College of Paediatrics and Child Health, London, UK, 2019, https://www. rcpch.ac.uk/work-we-do/bpsu/study-herpes-simplex-virus.

[4] National Institute for Health and Care Excellence, "Mersey Burns for calculating fluid resuscitation volume when managing burns," National Institute for Health and Care Excellence, London, UK, March 2016, https://www.nice.org.uk/advice/mib58/ resources/mersey-burns-for-calculating-fluid-resuscitationvolume-when-managing-burns-pdf-63499233245893.

[5] Nottingham Neonatal Service-Clinical Guidelines, Neonatal Thrombocytopenia, Nottingham Neonatal Service, Nottingham, UK, 2016, https://www.nuh.nhs.uk/download. $\mathrm{cfm}$ ?doc $=$ docm93jijm4n941.pdf\&ver=5045.

[6] Nottingham Neonatal Service-Clinical Guidelines, Red Cell Transfusion, Nottingham Neonatal Service, Nottingham, UK, https://www.nuh.nhs.uk/download.cfm?doc=docm 93j ijm4n4966.pdf\&ver=12451.

[7] Nottingham Neonatal Service-Clinical Guidelines, "Pain assessment and management on the neonatal intensive care unit," Nottingham Neonatal Service, Nottingham, UK, https://www.nuh. nhs.uk/download.cfm?doc=docm93jiim4n5147.pdf\&ver=10539.

[8] M. Avgil and A. Ornoy, "Herpes simplex virus and EpsteinBarr virus infections in pregnancy: consequences of neonatal or intrauterine infection," Reproductive Toxicology, vol. 21, no. 4, pp. 436-445, 2006.

[9] L. Marquez, M. L. Levy, F. M. Munoz, and D. L. Palazzi, "A report of three cases and review of intrauterine herpes simplex virus infection," The Pediatric Infectious Disease Journal, vol. 30, no. 2, pp. 153-157, 2011.

[10] Birmingham Women's and Children's NHS Foundation Trust, "Care of new-born baby with Epidermolysis Bullosa," Birmingham Women's and Children's NHS Foundation Trust, Birmingham, England, 2019, https://bwc.nhs.uk/ download.cfm?doc=docm93jijm4n1253.pdf\&ver $=4140$. 


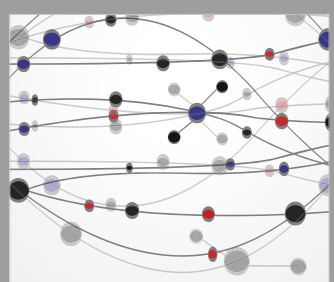

The Scientific World Journal
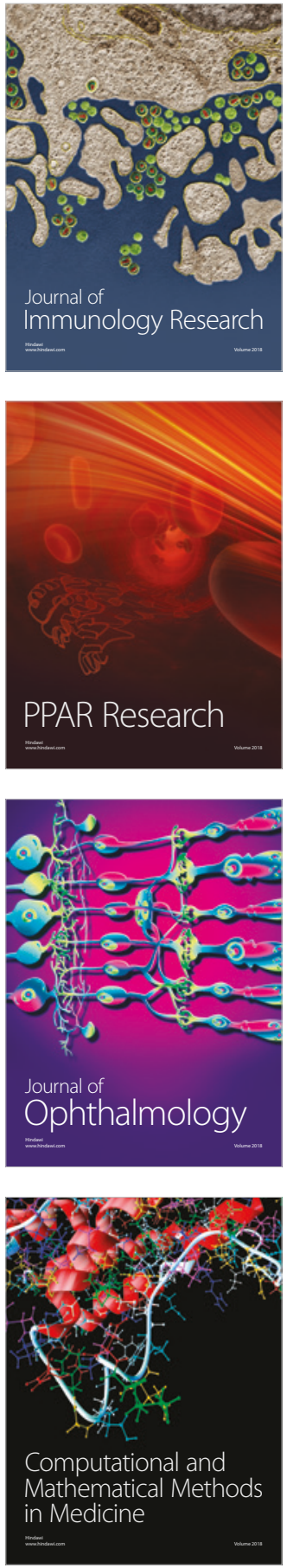

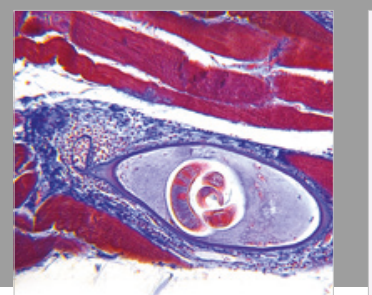

Gastroenterology Research and Practice

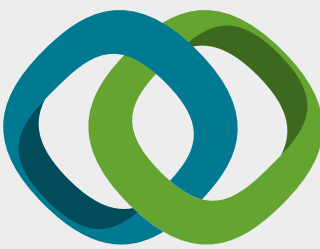

\section{Hindawi}

Submit your manuscripts at

www.hindawi.com
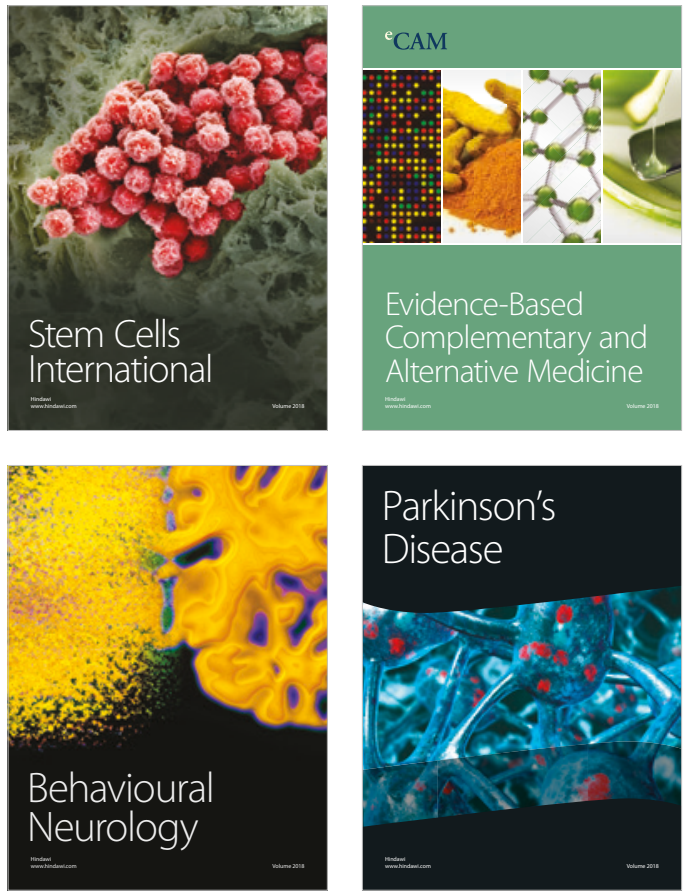

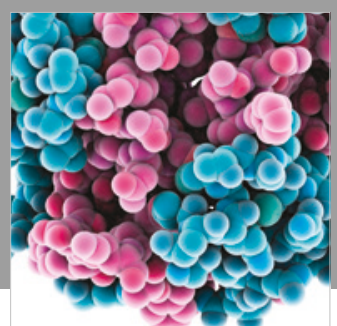

ournal of

Diabetes Research

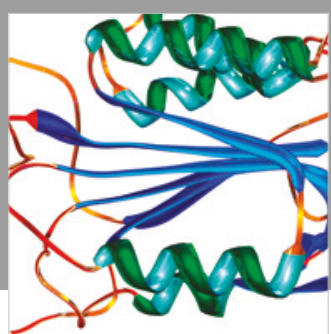

Disease Markers
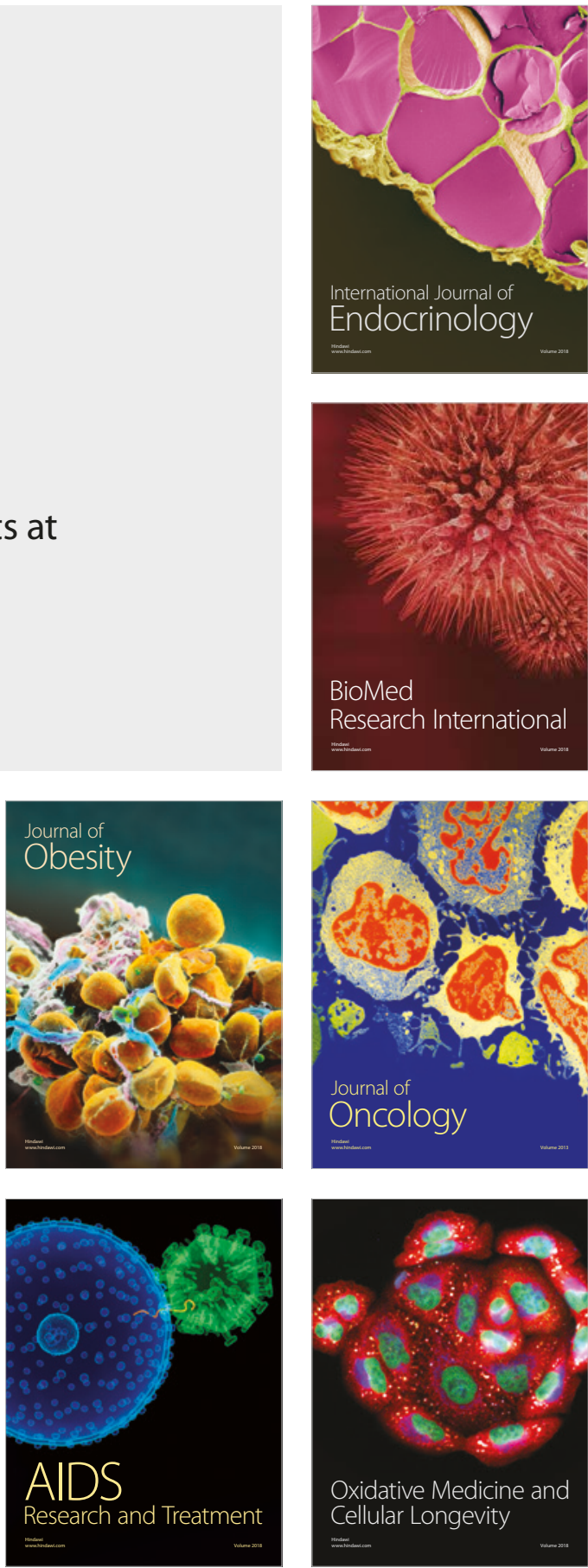